\title{
A Multidimensional Scaling Framework for Mobile Location Using Time-of-Arrival Measurements
}

\author{
K. W. Cheung and H. C. So, Member, IEEE
}

\begin{abstract}
Localization of mobile phones is now a very popular research topic. In this paper, a simple algorithm is devised for mobile location estimation using time-of-arrival measurements of the signal from the mobile station received at three or more base stations, via modifying the classical multidimensional scaling technique, which has been developed for analyzing data obtained from physical, biological, and behavioral science. The bias and variance of the proposed algorithm are also derived. Computer simulations are included to corroborate the theoretical development and to contrast the estimator performance with several conventional approaches as well as the Cramér-Rao lower bound.
\end{abstract}

Index Terms-Mobile terminal, multidimensional scaling, positioning algorithm, time-of-arrival.

\section{INTRODUCTION}

$\mathbf{T}$ HE problem of locating a mobile station (MS) has been receiving considerable interest, especially after the Federal Communications Commission (FCC) in the United States has adopted rules to improve the Emergency 911 (E-911) services by mandating the accuracy of locating a E-911 caller to be within a specified range, even for a wireless phone user [1]. Apart from E-911, mobile location services have triggered a large number of innovative applications [2]-[6]. For example, mobile position information is useful in monitoring the mentally impaired (e.g., elderly with Alzheimers disease), young children, and parolees. Moreover, logistics and bus companies can utilize this technology for fleet management to increase their efficiency. Other applications include location billing, interactive map consultation, and location-dependent advertising.

Global positioning system(GPS) can be used to provide mobile location. However, installing an additional GPS receiver to the MS will increase the power consumption and computational complexity, which ultimately impact the talk and standby time, size, weight, as well as cost of the MS [7]. Alternatively, the base stations (BSs) in the existing wireless network can be utilized to intercept the MS signal for position estimation. As a result, it is expected that mobile location using the BSs, instead of a GPS, is preferable and more cost-effective for the consumer.

Manuscript received August 4, 2003; revised February 19, 2004. This work was supported by a grant from the Research Grants Council of the Hong Kong Special Administrative Region, China, under Project CityU 1119/01E. The associate editor coordinating the review of this paper and approving it for publication was Dr. Fulvio Gini.

The authors are with the Department of Computer Engineering and Information Technology, City University of Hong Kong, Hong Kong (e-mail: gary.cheung@student.cityu.edu.hk; ithcso@ cityu.edu.hk).

Digital Object Identifier 10.1109/TSP.2004.840721
Common location approaches [2], [6] using the BSs are based on time-of-arrival (TOA), received signal strength (RSS), time-difference-of-arrival (TDOA), and/or angle-of-arrival (AOA) measurements determined from the MS signal received at the BSs. In this paper, we focus on two-dimensional mobile positioning given the TOA information. In the TOA method, the one-way propagation time of the signal traveling between the MS and each of the BSs is measured, which means that precisely synchronized clocks for the MS and all BSs are required, although such synchronization will not be needed if the round-trip time or two-way TOA is measured [2]. (Notice that for TDOA-based location systems, only synchronicity of the BSs is necessary because they use differences in TOAs rather than absolute time measurements as TOA-based location systems do.) Each TOA measurement then provides a circle centered at the BS on which the MS must lie. With three or more BSs, the measurements are converted into a set of circular equations, from which the MS position can be determined with the knowledge of the BS geometry. It should be pointed out that in practical situations, multipath propagation and nonline-of-sight (NLOS) propagation are two major sources of error, which can introduce large biases in the TOA measurements and result in unreliable MS position estimation. In fact, mitigation of the impairments due to multipath and/or NLOS is another key research topic in wireless location and recent works [8]-[12] in this area have reported some promising results. As a result, we reasonably assume that the multipath and NLOS errors in the TOA measurements have been successfully mitigated. It is worthy to note that accurate position estimation performance can be attained for location systems employing ultra-wideband signals [13], even when the multipath and NLOS errors are neglected.

There are basically two approaches for mobile positioning using TOA measurements. The first approach involves solving the nonlinear equations relating these measurements iteratively and commonly used techniques include linearization via Taylorseries expansion [14], [15], steepest descent method [16], and Newton-type iteration [17]. Although this approach can attain optimum estimation performance, it is computationally intensive and sufficiently precise initial estimates are required to obtain the global solution. On the other hand, the second approach, which allows real-time realization as well as ensures global convergence, reorganizes the nonlinear equations into a set of linear equations by introducing an extra variable that is a function of the source position. The linear equations can then be solved straightforwardly by using least squares (LS) as in the spherical interpolation (SI) technique [18], [19] for TDOA-based location systems, and the corresponding estimator is referred to as the 
least squares calibration (LSC) method [20] or by eliminating the common variable via subtraction of each equation from all others, which is referred to as the linear least squares (LLS) estimator [21]. We can also exploit the relation between the extra variable and MS position by using the two-step weighted least squares (WLS) through simple modification to [22], which is originated in location with TDOA measurements, or by employing the technique of Lagrange multipliers, which is referred to as the quadratic least squares (QLS) estimator [21]. Inspired by Birchfield's work [23] of utilizing classical multidimensional scaling (MDS) in microphone array calibration, in this paper, we derive a classical MDS approach for MS positioning using TOA measurements, which also provides a fast and globally convergence solution. Another similar and recent work on applying classical MDS for localization can be found in [24], where multiple nodes in a sensor network are needed to locate but only a few anchor nodes with known positions are available. Notice that there are many branches in MDS, and the location methods suggested in [16] and [17] in fact belong to the branch of the metric LS MDS [25].

Basically, MDS [25]-[27] is a field of study concerned with the search for a low-dimensional space in which points in the space represent the objects such that the distances, or dissimilarities, between the points in the space match. MDS has been a common technique for analyzing experimental data in physical, biological, and behavioral science. Classical MDS is a subset of MDS where the dissimilarities are Euclidean distances, which can be used to determine the relative coordinates of points given only their pairwise distances. By the same principle, it is expected that the MS location can be determined if the distances among the MS and BSs are provided. Our major contributions in this paper include i) development of an accurate and computationally attractive algorithm for mobile location via modifying the classical MDS technique (The idea is novel in the sense that the classical MDS is utilized although the computationally demanding metric LS MDS has been suggested in the literature [16], [17].); ii) investigation of the estimator relationship with the LSC, LLS, two-step WLS, and QLS location algorithms via consideration of the classical MDS method as an LS cost function minimization problem; iii) derivation of the bias and variance for the proposed algorithm, although most MDS analyzes do not include standard errors on parameter estimates [27]. Moreover, the derived bias and variance formulae can be used to produce the performance of the LSC, LLS, two-step WLS, and QLS estimators.

The rest of this paper is organized as follows. In Section II, we first present the model for the TOA measurements. Classical MDS is introduced via the development of an infeasible positioning algorithm. By modifying the classical technique, a practical mobile location algorithm is then derived. In Section III, prior to studying the bias and variance of the proposed method, a variance analysis formula for general optimization problems is developed. The relationships between the modified MDS, LSC, LLS, two-step WLS, and QLS methods are also examined. Simulation results are included in Section IV to evaluate the location estimator performance by comparison with the LLS, two-step WLS, and QLS estimators and to verify our theoretical findings. Finally, conclusions are drawn in Section V.

\section{TOA MEASUREMENT Model AND Positioning ALGORITHM DEVELOPMENT}

\section{A. TOA Measurement Model}

Let $\mathbf{x}_{0}=\left[\begin{array}{ll}x_{0} & y_{0}\end{array}\right]^{T}$ be the MS position to be determined and the known coordinates of the $i$ th BS be $\mathbf{x}_{i}=\left[\begin{array}{ll}x_{i} & y_{i}\end{array}\right]^{T}, i=$ $1,2, \ldots, M$, where $T$ denotes the transpose operation, and $M \geq$ 3 is the total number of receiving BSs. The distance between the $i$ th $\mathrm{BS}$ and $j$ th BS, which is denoted by $d_{i j}$, is given by

$$
\begin{gathered}
d_{i j}=\sqrt{\left(\mathbf{x}_{i}-\mathbf{x}_{j}\right)^{T}\left(\mathbf{x}_{i}-\mathbf{x}_{j}\right)}=\sqrt{\left(x_{i}-x_{j}\right)^{2}+\left(y_{i}-y_{j}\right)^{2}} \\
i=1,2, \ldots, M, \quad j=1,2, \ldots, M
\end{gathered}
$$

Similarly, the distance between the MS and the $i$ th BS is defined as

$$
\begin{aligned}
d_{i} & =d_{0 i}=d_{i 0}=\sqrt{\left(\mathbf{x}_{0}-\mathbf{x}_{i}\right)^{T}\left(\mathbf{x}_{0}-\mathbf{x}_{i}\right)} \\
& =\sqrt{\left(x_{0}-x_{i}\right)^{2}+\left(y_{0}-y_{i}\right)^{2}}, \quad i=1,2, \ldots, M .
\end{aligned}
$$

Since TOA is the one-way propagation time taken for the signal to travel from the MS to a BS, we have the following relationship:

$$
t_{i}=\frac{d_{i}}{c}, \quad i=1,2, \ldots, M
$$

where $t_{i}$ denotes the noise-free TOA at the $i$ th $\mathrm{BS}$, and $c$ is the speed of light. The range measurement based on $t_{i}$, in the presence of measurement errors denoted by $r_{i}$, is modeled as

$r_{i}=d_{i}+n_{i}=\sqrt{\left(x_{0}-x_{i}\right)^{2}+\left(y_{0}-y_{i}\right)^{2}}+n_{i}, \quad i=1,2, \ldots, M$

where $n_{i}$ is the range error in $r_{i}$. In order to facilitate the development and analysis of the proposed algorithm, it is assumed in our study that $\left\{n_{i}\right\}$ have zero means, although it is a simplification in real situations owing to multipath and NLOS propagation.

\section{B. Positioning Algorithm via Direct Use of MDS}

Classical MDS was first introduced in the discipline of psychology [28], [29], and its basic idea is to assume that the dissimilarities between objects are distances and then find coordinates that explain them. In particular, classical MDS can be used to solve the mathematical problem as follows: Given noisy distances between a set of points in a Euclidean space, estimate the coordinates of those points. In the following, we will develop a TOA-based positioning algorithm via direct application of the basic MDS algorithm [25]. Although the solution is not feasible, we can briefly see the know-how of MDS.

When employing MDS, it is assumed that the centroid of all the coordinates is at the origin, that is, $\sum_{i=0}^{M} \mathbf{x}_{i}=\left[\begin{array}{ll}0 & 0]^{T} \text {, which }\end{array}\right.$ means that a simple translation is required after the coordinates are recovered from the distance measurements. Given the distances among the MS and BSs, we first construct a matrix of 
squared distances, which are denoted by $\mathbf{D}$, as

$$
\mathbf{D}=\left[\begin{array}{ccccc}
0 & r_{1}^{2} & r_{2}^{2} & \cdots & r_{M}^{2} \\
r_{1}^{2} & 0 & d_{12}^{2} & \cdots & d_{1 M}^{2} \\
r_{2}^{2} & d_{21}^{2} & 0 & \cdots & d_{2 M}^{2} \\
\vdots & \vdots & \vdots & \ddots & \vdots \\
r_{M}^{2} & d_{M 1}^{2} & d_{M 2}^{2} & \cdots & 0
\end{array}\right]
$$

where the distances between the BSs are free of errors, but those involving the MS are noisy. Double centering is then applied to $\mathbf{D}$ to obtain a scalar product matrix, which is denoted by $\mathbf{B}$ and is of the form

where

$$
\mathbf{B}=-\frac{1}{2} \mathbf{J}_{M+1} \mathbf{D} \mathbf{J}_{M+1}
$$

$$
\mathbf{J}_{M+1}=\mathbf{I}_{M+1}-\frac{1}{M+1} \mathbf{1}_{M+1} \mathbf{1}_{M+1}^{T}
$$

is the centering matrix, with $\mathbf{I}_{M+1}$ and $\mathbf{1}_{M+1}$ denoting the $(M+$ $1) \times(M+1)$ identity matrix and $(M+1) \times 1$ column vector of all ones, respectively.

In the absence of distance errors such that $r_{i}=d_{i}, i=$ $1,2, \cdots, M$, it can be shown that

$$
\mathbf{B}=\mathbf{X} \mathbf{X}^{T}
$$

where $\mathbf{X}=\left[\begin{array}{llll}\mathbf{x}_{0} & \mathbf{x}_{1} & \cdots & \mathbf{x}_{M}\end{array}\right]^{T}$ is the matrix of coordinates with dimension $(M+1) \times 2$, or

$$
\begin{aligned}
{[\mathbf{B}]_{i j}=\mathbf{x}_{i}^{T} \mathbf{x}_{j} } & =-\frac{1}{2}\left(d_{i j}^{2}-\frac{1}{M+1} \sum_{i=0}^{M} d_{i j}^{2}\right. \\
& \left.-\frac{1}{M+1} \sum_{j=0}^{M} d_{i j}^{2}+\frac{1}{(M+1)^{2}} \sum_{i=0}^{M} \sum_{j=0}^{M} d_{i j}^{2}\right)
\end{aligned}
$$

which implies that the rank of $\mathbf{B}$ is 2 . Since $\mathbf{B}$ is symmetric and positive semi-definite, $\mathbf{B}$ can be decomposed using eigenvalue factorization as

$$
\mathbf{B}=\mathbf{V} \Lambda \mathbf{V}^{T}
$$

where $\boldsymbol{\Lambda}=\operatorname{diag}\left(\lambda_{1}, \lambda_{2}, \ldots, \lambda_{M+1}\right)$ is the diagonal matrix of eigenvalues of $\mathbf{B}$ with $\lambda_{1} \geq \lambda_{2} \geq \cdots \geq \lambda_{M+1} \geq 0$, and $\mathbf{V}=$ $\left[\begin{array}{llll}\mathbf{v}_{1} & \mathbf{v}_{2} & \cdots & \mathbf{v}_{M+1}\end{array}\right]$ is an orthonormal matrix whose columns are the corresponding eigenvectors. Since $\operatorname{rank}(\mathbf{B})=2$, we have $\lambda_{3}=\lambda_{4}=\cdots=\lambda_{M+1}=0$, and as a result, $\mathbf{B}$ can also be represented as

$$
\mathbf{B}=\mathbf{V}_{2} \boldsymbol{\Lambda}_{2} \mathbf{V}_{2}^{T}=\left(\mathbf{V}_{2} \boldsymbol{\Omega}\right) \boldsymbol{\Lambda}_{2}\left(\mathbf{V}_{2} \boldsymbol{\Omega}\right)^{T}
$$

where $\boldsymbol{\Lambda}_{2}=\operatorname{diag}\left(\lambda_{1}, \lambda_{2}\right), \mathbf{V}_{2}=\left[\mathbf{v}_{1} \mathbf{v}_{2}\right]$, and $\boldsymbol{\Omega}$ is an arbitrary orthogonal matrix. From (7) and (10), we get the so-called principle axes solution:

$$
\mathbf{X}^{\mathbf{r}}=\mathbf{V}_{2} \Lambda_{2}^{1 / 2}
$$

where $\Lambda_{2}^{1 / 2}=\operatorname{diag}\left(\lambda_{1}^{1 / 2}, \lambda_{2}^{1 / 2}\right)$.

In the practical situations of nonzero range errors, $\mathbf{B}$ will not be of rank 2 but has full rank. Nevertheless, (11) still yields an optimal estimate of $\mathbf{X}^{\mathbf{r}}$ in the LS sense because it has been proved [30] that the classical scaling solution is essentially

$$
\hat{\mathbf{X}}^{\mathbf{r}}=\arg \min _{\breve{\mathbf{X}}}\left\|\mathbf{B}-\breve{\mathbf{X}} \breve{\mathbf{X}}^{T}\right\|^{2}
$$

where $\breve{\mathbf{X}}$ is a matrix of variables, $\hat{\mathbf{X}}^{\mathbf{r}}$ is the corresponding coordinate matrix estimate, and $\|||$ represents the Frobenius norm. Obviously, the principle axes solution only estimates the coordinates of $\mathbf{X}$ up to an arbitrary rotation, and the requisite transformation matrix is given by [23]

$$
\boldsymbol{\Omega}=\left(\hat{\mathbf{X}}^{\mathbf{r} T} \mathbf{X} \mathbf{X}^{T} \hat{\mathbf{X}}^{\mathbf{r}}\right)^{\frac{1}{2}}\left(\mathbf{X}^{T} \hat{\mathbf{X}}^{\mathbf{r}}\right)^{-1} .
$$

The MS position estimate up to a translation is then

$$
\hat{\mathbf{x}}_{0}=\Omega^{T} \hat{\mathbf{x}}_{0}^{\mathbf{r}}
$$

where $\hat{\mathbf{x}}_{0}^{\mathbf{r}}$ corresponds to the first row of $\hat{\mathbf{x}}^{\mathbf{r}}$. Finally, performing proper translation based on the centroid assumption yields the estimate of $\mathbf{x}_{0}$.

However, from the above development of direct use of classical MDS for mobile positioning, we see that the basic algorithm is neither feasible nor reliable for the following reasons.

R1) The rotation matrix of (13) that requires $\mathbf{x}_{0}$, which is to be determined, is not known a priori.

R2) Even if an initial estimate of $\mathbf{x}_{0}$ is available, the rotation matrix of (13) is erroneous due to the noise in $\hat{\mathbf{X}}^{\mathbf{r}}$ as well as the MS position estimate. Note that although only the first column and row of $\mathbf{D}$ consist of measurement errors, all the elements in B will become noisy, which can be observed from (6), and hence, all the elements in $\hat{\mathbf{X}}^{\mathbf{r}}$ consist of noise as well. Furthermore, error is introduced in performing the translation in the final step based on the estimate of $\mathbf{x}_{0}$. As a result, it is not expected that the resultant MS position estimate is reliable.

\section{Proposed Positioning Algorithm via Modifying MDS}

In this section, a modified MDS algorithm is proposed for mobile positioning so that the problems in R1) and R2) are overcome. The key idea is to change the centroid requirement by excluding the unknown MS coordinates, that is, $\sum_{i=1}^{M} \mathbf{x}_{i}=$ $\left[\begin{array}{ll}0 & 0\end{array}\right]^{T}$. It is noteworthy that this centroid requirement is identical to the centroid centered reference system assumption used in [21]. In so doing, the corresponding transformation matrix, which is denoted by $\boldsymbol{\Omega}^{\prime}$, will not involve $\mathbf{x}_{0}$, which implies the feasibility of the algorithm. Moreover, the corresponding translation vector will be free of error. Our second improvement attempts to remove the noise in $\boldsymbol{\Omega}^{\prime}$ by utilizing the known information of the BS positions in order to give a perfect rotation matrix, if possible.

Since the centroid of all the coordinates is not at the origin now, the proposed algorithm has to be derived from the first principle of classical MDS as follows. First of all, we express (1) and (2) using vector notation:

$$
\begin{aligned}
& d_{i j}^{2}=\mathbf{x}_{i}^{T} \mathbf{x}_{i}+\mathbf{x}_{j}^{T} \mathbf{x}_{j}-2 \mathbf{x}_{i}^{T} \mathbf{x}_{j} \\
& i=0,1, \cdots, M, \quad j=0,1, \cdots, M
\end{aligned}
$$


From (15), we have

$$
\sum_{i=0}^{M} d_{i j}^{2}=\sum_{i=0}^{M} \mathbf{x}_{i}^{T} \mathbf{x}_{i}+(M+1) \mathbf{x}_{j}^{T} \mathbf{x}_{j}-2 \sum_{i=0}^{M} \mathbf{x}_{i}^{T} \mathbf{x}_{j}
$$

Dividing both sides of (16) by $(M+1)$ and with the use of $\sum_{i=0}^{M} \mathbf{x}_{i}=\mathbf{x}_{0}$ gives

$$
\begin{array}{r}
\frac{1}{M+1} \sum_{i=0}^{M} d_{i j}^{2}=\frac{1}{M+1} \sum_{i=0}^{M} \mathbf{x}_{i}^{T} \mathbf{x}_{i}+\mathbf{x}_{j}^{T} \mathbf{x}_{j}-\frac{2}{M+1} \mathbf{x}_{0}^{T} \mathbf{x}_{j} \\
j=0,1, \cdots, M
\end{array}
$$

Interchanging the indexes of $i$ and $j$ in (17), we get another equation

$$
\begin{array}{r}
\frac{1}{M+1} \sum_{j=0}^{M} d_{i j}^{2}=\mathbf{x}_{i}^{T} \mathbf{x}_{i}+\frac{1}{M+1} \sum_{j=0}^{M} \mathbf{x}_{j}^{T} \mathbf{x}_{j}-\frac{2}{M+1} \mathbf{x}_{i}^{T} \mathbf{x}_{0} \\
i=0,1, \cdots, M
\end{array}
$$

Summing up all $(M+1)$ equations of (18) and then dividing the resultant equation by $(M+1)$ yields

$$
\frac{1}{(M+1)^{2}} \sum_{i=0}^{M} \sum_{j=0}^{M} d_{i j}^{2}=\frac{2}{M+1} \sum_{i=0}^{M} \mathbf{x}_{i}^{T} \mathbf{x}_{i}-\frac{2}{(M+1)^{2}} \mathbf{x}_{0}^{T} \mathbf{x}_{0} .
$$

Substituting (17)-(19) into (15), the term $\mathbf{x}_{i}^{T} \mathbf{x}_{j}$ is expressed as

$$
\begin{aligned}
\mathbf{x}_{i}^{T} \mathbf{x}_{j}=-\frac{1}{2}\left(d_{i j}^{2}-\frac{1}{M+1} \sum_{i=0}^{M} d_{i j}^{2}-\frac{1}{M+1} \sum_{j=0}^{M} d_{i j}^{2}\right. \\
\left.\quad+\frac{1}{(M+1)^{2}} \sum_{i=0}^{M} \sum_{j=0}^{M} d_{i j}^{2}\right)+\frac{1}{M+1} \mathbf{x}_{0}^{T} \mathbf{x}_{j} \\
+\frac{1}{M+1} \mathbf{x}_{i}^{T} \mathbf{x}_{0}-\frac{1}{(M+1)^{2}} \mathbf{x}_{0}^{T} \mathbf{x}_{0}
\end{aligned}
$$

Notice that (20) differs from (8) because of different centroid assumptions employed. From (8) and (20) and the known BS positions, we are going to construct a modified scalar product matrix denoted by $\mathbf{B}^{\prime}$, which agrees with the new centroid assumption. First, we partition $\mathbf{B}$ in (6) as

$$
\mathbf{B}=\left[\begin{array}{cc}
B_{11} & \mathbf{b}^{T} \\
\mathbf{b} & \mathbf{B}_{1}
\end{array}\right]
$$

where $B_{11}$ is the first diagonal element of $\mathbf{B}, \mathbf{b}$ is an $M \times 1$ column vector, and $\mathbf{B}_{1}$ is an $M \times M$ matrix. Similarly, $\mathbf{B}^{\prime}$ is partitioned as

$$
\mathbf{B}^{\prime}=\left[\begin{array}{cc}
B_{11}^{\prime} & \mathbf{b}^{\prime T} \\
\mathbf{b}^{\prime} & \mathbf{B}_{1}^{\prime}
\end{array}\right]
$$

where $B_{11}^{\prime}, \mathbf{b}^{\prime}$, and $\mathbf{B}_{1}^{\prime}$ are to be determined. It is obvious that the matrix $\mathbf{B}_{1}^{\prime}$ can be evaluated as

$$
\mathbf{B}_{1}^{\prime}=\mathbf{X}_{\mathrm{BS}} \mathbf{X}_{\mathrm{BS}}^{T}
$$

where

$$
\mathbf{X}_{\mathrm{BS}}=\left[\mathbf{x}_{1} \mathbf{x}_{2} \cdots \mathbf{x}_{M}\right]^{T}
$$

which is free of measurement error. Using (20), the values of $\mathbf{x}_{i}^{T} \mathbf{x}_{0}, \mathbf{x}_{0}^{T} \mathbf{x}_{j}$, and $\mathbf{x}_{0}^{T} \mathbf{x}_{0}$ are computed as

$$
\begin{aligned}
\mathbf{x}_{i}^{T} \mathbf{x}_{0}= & -\frac{1}{2}\left(\frac{M+1}{M}\right) \\
& \times\left(d_{i}^{2}-\frac{1}{M+1} \sum_{i=0}^{M} d_{i}^{2}-\frac{1}{M+1} \sum_{j=0}^{M} d_{i j}^{2}\right. \\
& \left.+\frac{1}{(M+1)^{2}} \sum_{i=0}^{M} \sum_{j=0}^{M} d_{i j}^{2}\right)+\frac{1}{M+1} \mathbf{x}_{0}^{T} \mathbf{x}_{0} \\
\mathbf{x}_{0}^{T} \mathbf{x}_{j}= & -\frac{1}{2}\left(\frac{M+1}{M}\right) \\
\times & \left(d_{j}^{2}-\frac{1}{M+1} \sum_{i=0}^{M} d_{i j}^{2}-\frac{1}{M+1} \sum_{j=0}^{M} d_{j}^{2}\right. \\
& \left.+\frac{1}{(M+1)^{2}} \sum_{i=0}^{M} \sum_{j=0}^{M} d_{i j}^{2}\right)+\frac{1}{M+1} \mathbf{x}_{0}^{T} \mathbf{x}_{0} \\
j= & 1,2, \cdots, M
\end{aligned}
$$

and

$$
\begin{aligned}
\mathbf{x}_{0}^{T} \mathbf{x}_{0}=-\frac{1}{2}\left(\frac{M+1}{M}\right)^{2}( & -\frac{2}{M+1} \sum_{i=0}^{M} d_{i}^{2} \\
& \left.+\frac{1}{(M+1)^{2}} \sum_{i=0}^{M} \sum_{j=0}^{M} d_{i j}^{2}\right)
\end{aligned}
$$

By comparing (24)-(26) and the corresponding terms based on (8), the relationships between $B_{11}$ and $B_{11}^{\prime}$, as well as $\mathbf{b}$ and $\mathbf{b}^{\prime}$, are found. As a result, $\mathbf{B}^{\prime}$ is constructed as

$\mathbf{B}^{\prime}=\left[\begin{array}{cc}\left(\frac{M+1}{M}\right)^{2} B_{11} & \left(\frac{M+1}{M}\right) \mathbf{b}^{T}+\left(\frac{M+1}{M^{2}}\right) B_{11} \mathbf{1}_{M}^{T} \\ \left(\frac{M+1}{M}\right) \mathbf{b}+\left(\frac{M+1}{M^{2}}\right) B_{11} \mathbf{1}_{M} & \mathbf{X}_{\mathrm{BS}} \mathbf{X}_{\mathrm{BS}}^{T}\end{array}\right]$

With the use of $\mathbf{B}^{\prime}$, the MS position can be estimated in a manner similar to the basic MDS algorithm. The procedure of our proposed positioning algorithm via modifying the classical MDS technique is presented as follows.

i) Compute $B_{11}$ and $\mathbf{b}$ in (21) as well as $\mathbf{B}_{1}^{\prime}$ in (23).

ii) Construct $\mathbf{B}^{\prime}$ using (27).

iii) Decompose $\mathbf{B}^{\prime}$ as $\mathbf{B}^{\prime}=\mathbf{V}^{\prime} \Lambda^{\prime} \mathbf{V}^{\prime T}$, where $\Lambda^{\prime}=\operatorname{diag}$ $\left(\lambda_{1}^{\prime}, \lambda_{2}^{\prime}, \ldots, \lambda_{M+1}^{\prime}\right)$ with $\lambda_{1}^{\prime} \geq \lambda_{2}^{\prime} \geq \cdots \geq \lambda_{M+1}^{\prime} \geq$ 0 , and $\mathbf{V}^{\prime}=\left[\mathbf{v}_{1}^{\prime} \mathbf{v}_{2}^{\prime} \cdots \mathbf{v}_{M+1}^{\prime}\right]$.

iv) Compute the principle axes solution as $\hat{\mathbf{X}}^{\mathbf{r}}=\mathbf{V}_{2}^{\prime}$ $\Lambda_{2}^{\prime(1 / 2)}$, where $\mathbf{V}_{2}^{\prime}=\left[\mathbf{v}_{1}^{\prime} \mathbf{v}_{2}^{\prime}\right]$, and $\Lambda_{2}^{\prime(1 / 2)}=\operatorname{diag}$ $\left(\lambda_{1}^{\prime(1 / 2)}, \lambda_{2}^{\prime(1 / 2)}\right)$.

v) Partition $\hat{\mathbf{X}}^{\mathbf{r}}$ as $\hat{\mathbf{X}}^{\mathbf{r}}=\left[\hat{\mathbf{x}}_{0}^{\mathbf{r}} \hat{\mathbf{X}}_{\mathrm{BS}}^{\mathbf{r} T}\right]^{T}$, where $\hat{\mathbf{x}}_{0}^{\mathbf{r}}$ and $\hat{\mathbf{X}}_{\mathrm{BS}}^{\mathbf{r}}$ correspond to the rotated coordinates of the MS and 
BSs, respectively. Following [23], the requisite rotation matrix for $\sum_{i=1}^{M} \mathbf{x}_{i}=\left[\begin{array}{ll}0 & 0\end{array}\right]^{T}$ is determined as

$$
\mathbf{\Omega}^{\prime}=\left(\hat{\mathbf{X}}_{\mathrm{BS}}^{\mathrm{rT}} \mathbf{X}_{\mathrm{BS}} \mathbf{X}_{\mathrm{BS}}^{T} \hat{\mathbf{X}}_{\mathrm{BS}}^{\mathbf{r}}\right)^{\frac{1}{2}}\left(\mathbf{X}_{\mathrm{BS}}^{T} \hat{\mathbf{X}}_{\mathrm{BS}}^{\mathbf{r}}\right)^{-1} \text {. }
$$

We expect that there is insignificant difference between $\mathbf{B}_{1}^{\prime}$ and the corresponding partitioned matrix determined from the truncated eigedecomposition in (10) because $\mathbf{B}_{1}^{\prime}$ consists of no errors; hence, the noise in the resultant rotated matrix of $\mathbf{X}_{\mathrm{BS}}$ based on the principle axes solution will become negligible. As a result, the rotation matrix of (28) will be very accurate.

vi) Find the estimated MS position up to a translation using $\hat{\mathbf{x}}_{0}=\boldsymbol{\Omega}^{\prime T} \hat{\mathbf{x}}_{0}^{\mathbf{r}}$, as in (14).

vii) Perform proper translation using the known BS coordinates to get the MS position estimate.

We see that the proposed algorithm allows real-time computation and requires no initial estimate of the MS position for global convergence. It is also simple to implement and can be easily extended to three-dimensional mobile positioning.

\section{Performance Analysis}

In this section, the bias and variance of the MS position estimate in the proposed MDS algorithm will be studied. The basic idea is to consider that the algorithm is essentially solving a cost function minimization problem as in (12). We first develop a variance expression for general minimization or maximization problems where a parameter vector is to be estimated. Under sufficiently small range error conditions, it is proved that the MDS algorithm is unbiased, and the variance formula is then applied to produce its theoretical estimation performance. Finally, using the interpretation of cost function minimization, the relationships of the MDS method with the LSC, LLS, QLS, and two-step WLS estimators will be examined.

\section{A. Variance Expression for General Optimization Problems}

Let $J$ be a real cost function which is a continuous function of a number of variables, or a column variable vector, say, $\breve{\mathbf{x}}_{0}$. In a typical optimization process, we need to find an estimate of the parameter vector $\mathbf{x}_{0}$ denoted by $\hat{\mathbf{x}}_{0}$, which corresponds to the global minimum or maximum of $J$. This implies that

$$
\left.\frac{\partial J}{\partial \breve{\mathbf{x}}_{0}}\right|_{\breve{\mathbf{x}}_{0}=\hat{\mathbf{x}}_{0}}=\mathbf{0}
$$

where $\mathbf{0}$ denotes a column vector of zero elements with length equals to that of $\hat{\mathbf{x}}_{0}$. When $\hat{\mathbf{x}}_{0}$ is located at a reasonable proximity of the ideal solution of $\mathbf{x}_{0}$, using Taylor's series to expand $\left(\partial J / \partial \breve{\mathbf{x}}_{0}\right)$ around $\mathbf{x}_{0}$ up to the first-order terms, we have

$$
-\left.\left.\frac{\partial J}{\partial \breve{\mathbf{x}}_{0}}\right|_{\breve{\mathbf{x}}_{0}=\mathbf{x}_{0}} \approx \frac{\partial^{2} J}{\partial \breve{\mathbf{x}}_{0} \partial \breve{\mathbf{x}}_{0}^{T}}\right|_{\breve{\mathbf{x}}_{0}=\mathbf{x}_{0}}\left(\hat{\mathbf{x}}_{0}-\mathbf{x}_{0}\right)
$$

Note that $\left(\partial^{2} J\right) /\left(\partial \breve{\mathbf{x}}_{0} \partial \breve{\mathbf{x}}_{0}^{T}\right)$ is called the Hessian matrix of $J$, which is a symmetric matrix, that is, $\left(\partial^{2} J\right) /\left(\partial \breve{\mathbf{x}}_{0} \partial \breve{\mathbf{x}}_{0}^{T}\right)=$ $\left(\partial^{2} J / \partial \breve{\mathbf{x}}_{0} \partial \breve{\mathbf{x}}_{0}^{T}\right)^{T}$.
Assuming that the second derivatives inside the Hessian matrix of $J$ are smooth enough around $\mathbf{x}_{0}$, then (30) can be approximated as

$$
-\left.\left.\frac{\partial J}{\partial \breve{\mathbf{x}}_{0}}\right|_{\breve{\mathbf{x}}_{0}=\mathbf{x}_{0}} \approx E\left[\frac{\partial^{2} J}{\partial \breve{\mathbf{x}}_{0} \partial \breve{\mathbf{x}}_{0}^{T}}\right]\right|_{\breve{\mathbf{x}}_{0}=\mathbf{x}_{0}}\left(\hat{\mathbf{x}}_{0}-\mathbf{x}_{0}\right) .
$$

Taking the transpose on both sides of (31) gives

$$
-\left.\left.\left(\frac{\partial J}{\partial \breve{\mathbf{x}}_{0}}\right)^{T}\right|_{\breve{\mathbf{x}}_{0}=\mathbf{x}_{0}} \approx\left(\hat{\mathbf{x}}_{0}-\mathbf{x}_{0}\right)^{T} E\left[\frac{\partial^{2} J}{\partial \breve{\mathbf{x}}_{0} \partial \breve{\mathbf{x}}_{0}^{T}}\right]\right|_{\breve{\mathbf{x}}_{0}=\mathbf{x}_{0}} .
$$

Multiplying (31) by (32) and then taking the expected value yields

$$
\begin{gathered}
\left.\left.E\left[\left(\frac{\partial J}{\partial \breve{\mathbf{x}}_{0}}\right)\left(\frac{\partial J}{\partial \breve{\mathbf{x}}_{0}}\right)^{T}\right]\right|_{\breve{\mathbf{x}}_{0}=\mathbf{x}_{0}} \approx E\left[\frac{\partial^{2} J}{\partial \breve{\mathbf{x}}_{0} \partial \breve{\mathbf{x}}_{0}^{T}}\right]\right|_{\breve{\mathbf{x}}_{0}=\mathbf{x}_{0}} \\
\left.\cdot E\left[\left(\hat{\mathbf{x}}_{0}-\mathbf{x}_{0}\right)\left(\hat{\mathbf{x}}_{0}-\mathbf{x}_{0}\right)^{T}\right] \cdot E\left[\frac{\partial^{2} J}{\partial \breve{\mathbf{x}}_{0} \partial \breve{\mathbf{x}}_{0}^{T}}\right]\right|_{\breve{\mathbf{x}}_{0}=\mathbf{x}_{0}} .
\end{gathered}
$$

When the estimate of $\mathbf{x}_{0}$ is unbiased, that is, $E\left[\hat{\mathbf{x}}_{0}\right]=\mathbf{x}_{0}$, $E\left[\left(\hat{\mathbf{x}}_{0}-\mathbf{x}_{0}\right)\left(\hat{\mathbf{x}}_{0}-\mathbf{x}_{0}\right)^{T}\right]$ represents the covariance matrix of $\hat{\mathbf{x}}_{0}$, and we denote it by $\mathbf{C}_{\mathbf{x}_{0}}$. By rearranging (33), $\mathbf{C}_{\mathbf{x}_{0}}$ is computed as

$$
\begin{aligned}
\mathbf{C}_{x_{0}} \approx E\left[\frac{\partial^{2} J}{\partial \breve{\mathbf{x}}_{0} \partial \breve{\mathbf{x}}_{0}^{T}}\right]^{-1} E\left[\left(\frac{\partial J}{\partial \breve{\mathbf{x}}_{0}}\right)\left(\frac{\partial J}{\partial \breve{\mathbf{x}}_{0}}\right)^{T}\right] \\
\left.\cdot E\left[\frac{\partial^{2} J}{\partial \breve{\mathbf{x}}_{0} \partial \breve{\mathbf{x}}_{0}^{T}}\right]^{-1}\right|_{\breve{\mathbf{x}}_{0}=\mathbf{x}_{0}} .
\end{aligned}
$$

The variances of $\hat{\mathbf{x}}_{0}$ are then given by the diagonal elements of $\mathbf{C}_{\mathbf{x}_{0}}$. It is expected that (34) can predict the parameter estimation performance of minimizing or maximizing the cost function $J$ for sufficiently small noise conditions, according to our assumptions in the variance expression development.

\section{B. Bias and Variance for Proposed Algorithm}

Based on (12), the cost function to be minimized in the proposed method is

$$
J=\left\|\mathbf{B}^{\prime}-\breve{\mathbf{X}} \breve{\mathbf{X}}^{T}\right\|^{2}=\operatorname{tr}\left\{\left(\mathbf{B}^{\prime}-\breve{\mathbf{X}} \breve{\mathbf{X}}^{T}\right)\left(\mathbf{B}^{\prime}-\breve{\mathbf{X}} \breve{\mathbf{X}}^{T}\right)^{T}\right\}
$$

where tr denotes the trace operator. Since $\breve{\mathbf{X}}$ is unique up to a rotation, we can restrict it in the form of $\breve{\mathbf{X}}=\left[\begin{array}{llll}\mathbf{x}_{0} & \mathbf{x}_{1} & \cdots & \mathbf{x}_{M}\end{array}\right]$ so that $\breve{\mathbf{x}}_{0}=\left[\breve{x}_{0} \breve{y}_{0}\right]^{T}$ are the only variables. Now, we are going to represent (35) as a function of $\breve{\mathbf{x}}_{0}$.

First, it is noticed that $\mathbf{B}^{\prime}$ is equal to $\breve{\mathbf{X}} \breve{X}^{T}$ except for the elements in the first column and row. This means that when computing the error matrix $\left(\mathbf{B}^{\prime}-\breve{\mathbf{X}} \breve{\mathbf{X}}^{T}\right)$, only the errors in $\mathbf{x}_{i}^{T} \breve{\mathbf{x}}_{0}$, $\breve{\mathbf{x}}_{0}^{T} \mathbf{x}_{j}$, and $\breve{\mathbf{x}}_{0}^{T} \breve{\mathbf{x}}_{0}$ are required. Let the squared distance error for the $i$ th $\mathrm{BS}$ be

$$
\begin{aligned}
\varepsilon_{i}= & r_{i}^{2}-\left[\left(x_{i}-\breve{x}_{0}\right)^{2}+\left(y_{i}-\breve{y}_{0}\right)^{2}\right] \\
= & {\left[2 x_{i} \breve{x}_{0}+2 y_{i} \breve{y}_{0}-\left(\breve{x}_{0}^{2}+\breve{y}_{0}^{2}\right)\right]-\left[x_{i}^{2}+y_{i}^{2}-r_{i}^{2}\right] } \\
= & 2\left(\left[x_{i} \breve{x}_{0}+y_{i} \breve{y}_{0}-0.5\left(\breve{x}_{0}^{2}+\breve{y}_{0}^{2}\right)\right]-\frac{1}{2}\left[x_{i}^{2}+y_{i}^{2}-r_{i}^{2}\right]\right) \\
& i=1,2, \ldots, M
\end{aligned}
$$


From (24), the error in $\mathbf{x}_{i}^{T} \breve{\mathbf{x}}_{0}$ is observed as

$$
\begin{aligned}
\Delta \mathbf{x}_{i}^{T} \breve{\mathbf{x}}_{0}= & -\frac{1}{2}\left(\frac{M+1}{M}\right) \\
& \times\left(\varepsilon_{i}-\frac{1}{M+1} \sum_{j=1}^{M} \varepsilon_{j}-\frac{1}{M+1} \varepsilon_{i}\right. \\
& \left.+\frac{2}{(M+1)^{2}} \sum_{j=1}^{M} \varepsilon_{j}\right)+\frac{1}{M(M+1)} \sum_{j=1}^{M} \varepsilon_{j} \\
= & -\frac{1}{2} \varepsilon_{i}+\frac{1}{2 M} \sum_{j=1}^{M} \varepsilon_{j}, \quad i=1,2, \cdots, M .
\end{aligned}
$$

Similarly, from (25) and (26), the errors in $\breve{\mathbf{x}}_{0}^{T} \mathbf{x}_{j}$ and $\breve{\mathbf{x}}_{0}^{T} \breve{\mathbf{x}}_{0}$ are

$$
\Delta \breve{\mathbf{x}}_{0}^{T} \mathbf{x}_{j}=-\frac{1}{2} \varepsilon_{j}+\frac{1}{2 M} \sum_{i=1}^{M} \varepsilon_{i}, \quad j=1,2, \cdots, M
$$
and

$$
\Delta \breve{\mathbf{x}}_{0}^{T} \breve{\mathbf{x}}_{0}=\frac{1}{M} \sum_{i=1}^{M} \varepsilon_{i}
$$

Using (37)-(39), the error matrix is then expressed as

$$
\mathbf{B}^{\prime}-\breve{\mathbf{X}} \breve{\mathbf{X}}^{T}=\frac{1}{M} \sum_{i=1}^{M} \varepsilon_{i} \mathbf{P}-\frac{1}{2} \mathbf{Q}
$$

where

$$
\mathbf{P}=\left[\begin{array}{cccc}
1 & 0.5 & \cdots & 0.5 \\
0.5 & 0 & \cdots & 0 \\
\vdots & \vdots & \ddots & \vdots \\
0.5 & 0 & \cdots & 0
\end{array}\right]
$$

and

$$
\mathbf{Q}=\left[\begin{array}{cccc}
0 & \varepsilon_{1} & \cdots & \varepsilon_{M} \\
\varepsilon_{1} & 0 & \cdots & 0 \\
\vdots & \vdots & \ddots & \vdots \\
\varepsilon_{M} & 0 & \cdots & 0
\end{array}\right]
$$

Hence, we have

$$
\begin{aligned}
\left(\mathbf{B}^{\prime}-\breve{\mathbf{X}} \breve{\mathbf{X}}^{T}\right)\left(\mathbf{B}^{\prime}-\breve{\mathbf{X}} \breve{\mathbf{X}}^{T}\right)^{T} \\
=\left(\frac{1}{M} \sum_{i=1}^{M} \varepsilon_{i}\right)^{2} \mathbf{P} \mathbf{P}^{T}-\frac{1}{2 M} \sum_{i=1}^{M} \varepsilon_{i} \mathbf{Q P}^{T} \\
\quad-\frac{1}{2 M} \sum_{i=1}^{M} \varepsilon_{i} \mathbf{P} \mathbf{Q}^{T}+\frac{1}{4} \mathbf{Q} \mathbf{Q}^{T}
\end{aligned}
$$

Since

$$
\begin{aligned}
& \mathbf{P P}^{T}=\left[\begin{array}{cccc}
1 & 0.5 & \cdots & 0.5 \\
0.5 & 0 & \cdots & 0 \\
\vdots & \vdots & \ddots & \vdots \\
0.5 & 0 & \cdots & 0
\end{array}\right]\left[\begin{array}{cccc}
1 & 0.5 & \cdots & 0.5 \\
0.5 & 0 & \cdots & 0 \\
\vdots & \vdots & \ddots & \vdots \\
0.5 & 0 & \cdots & 0
\end{array}\right] \\
& =\left[\begin{array}{cccc}
1+0.25 M & * & \cdots & * \\
* & 0.25 & \cdots & * \\
\vdots & \vdots & \ddots & \vdots \\
* & * & \cdots & 0.25
\end{array}\right]
\end{aligned}
$$

$$
\begin{aligned}
& \mathbf{Q Q}^{T}=\left[\begin{array}{cccc}
0 & \varepsilon_{1} & \cdots & \varepsilon_{M} \\
\varepsilon_{1} & 0 & \cdots & 0 \\
\vdots & \vdots & \ddots & \vdots \\
\varepsilon_{M} & 0 & \cdots & 0
\end{array}\right]\left[\begin{array}{cccc}
0 & \varepsilon_{1} & \cdots & \varepsilon_{M} \\
\varepsilon_{1} & 0 & \cdots & 0 \\
\vdots & \vdots & \ddots & \vdots \\
\varepsilon_{M} & 0 & \cdots & 0
\end{array}\right] \\
& =\left[\begin{array}{cccc}
\sum_{i=1}^{M} \varepsilon_{i}^{2} & * & \cdots & * \\
* & \varepsilon_{i}^{2} & \cdots & * \\
\vdots & \vdots & \ddots & \vdots \\
* & * & \cdots & \varepsilon_{i}^{2}
\end{array}\right] \\
& \mathbf{Q P}^{T}=\left[\begin{array}{cccc}
0 & \varepsilon_{1} & \cdots & \varepsilon_{M} \\
\varepsilon_{1} & 0 & \cdots & 0 \\
\vdots & \vdots & \ddots & \vdots \\
\varepsilon_{M} & 0 & \cdots & 0
\end{array}\right]\left[\begin{array}{cccc}
1 & 0.5 & \cdots & 0.5 \\
0.5 & 0 & \cdots & 0 \\
\vdots & \vdots & \ddots & \vdots \\
0.5 & 0 & \cdots & 0
\end{array}\right] \\
& =\left[\begin{array}{cccc}
0.5 \sum_{i=1}^{M} \varepsilon_{i} & * & \cdots & * \\
* & \varepsilon_{i} & \cdots & * \\
\vdots & \vdots & \ddots & \vdots \\
* & * & \cdots & \varepsilon_{i}
\end{array}\right]
\end{aligned}
$$

and

$$
\begin{aligned}
\mathbf{P Q}^{T}= & {\left[\begin{array}{cccc}
1 & 0.5 & \cdots & 0.5 \\
0.5 & 0 & \cdots & 0 \\
\vdots & \vdots & \ddots & \vdots \\
0.5 & 0 & \cdots & 0
\end{array}\right]\left[\begin{array}{cccc}
0 & \varepsilon_{1} & \cdots & \varepsilon_{M} \\
\varepsilon_{1} & 0 & \cdots & 0 \\
\vdots & \vdots & \ddots & \vdots \\
\varepsilon_{M} & 0 & \cdots & 0
\end{array}\right] } \\
= & {\left[\begin{array}{cccc}
0.5 \sum_{i=1}^{M} \varepsilon_{i} & * & \cdots & * \\
* & \varepsilon_{i} & \cdots & * \\
\vdots & \vdots & \ddots & \vdots \\
* & * & \cdots & \varepsilon_{i}
\end{array}\right] }
\end{aligned}
$$

where $*$ represents the elements of no significance, substituting (42)-(45) into (41) and then taking its trace gives

$$
\begin{aligned}
J & =\operatorname{tr}\left\{\left(\mathbf{B}^{\prime}-\breve{\mathbf{X}} \breve{\mathbf{X}}^{T}\right)\left(\mathbf{B}^{\prime}-\breve{\mathbf{X}} \breve{\mathbf{X}}^{T}\right)^{T}\right\} \\
& =\left(\frac{1}{M} \sum_{i=1}^{M} \varepsilon_{i}\right)^{2}(1+0.5 M)-\frac{1}{M}\left(\sum_{i=1}^{M} \varepsilon_{i}\right)^{2}+\frac{1}{2} \sum_{i=1}^{M} \varepsilon_{i}^{2} \\
& =\left(\frac{1}{2}-\frac{M-2}{2 M^{2}}\right) \sum_{i=1}^{M} \varepsilon_{i}^{2}-2\left(\frac{M-2}{2 M^{2}}\right) \sum_{i=1}^{M} \sum_{j=1, j \neq i}^{M} \varepsilon_{i} \varepsilon_{j} .
\end{aligned}
$$

It is interesting to note that the first term of (46) consists of the sum of square errors, whereas the second term contributes to the cross-products of the errors, which means that $J$ is a generalized LS cost function. In order to utilize (34) to evaluate the theoretical performance of the proposed algorithm, $J$ has to be expressed in matrix-vector form as follows. Expressing the elements of (36) in a vector, we have

$$
\varepsilon=\left[\varepsilon_{1} \varepsilon_{2} \cdots \varepsilon_{M}\right]^{T}=2\left[\breve{G}_{0}-0.5\left(\breve{\mathbf{x}}_{0}^{T} \breve{\mathbf{x}}_{0}\right) \mathbf{1}_{M}-\mathbf{h}\right]
$$

where

$$
\mathbf{G}=\left[\begin{array}{cc}
x_{1} & y_{1} \\
x_{2} & y_{2} \\
\vdots & \vdots \\
x_{M} & y_{M}
\end{array}\right]
$$


and

$$
\mathbf{h}=\frac{1}{2}\left[\begin{array}{c}
x_{1}^{2}+y_{1}^{2}-r_{1}^{2} \\
x_{2}^{2}+y_{2}^{2}-r_{2}^{2} \\
\vdots \\
x_{M}^{2}+y_{M}^{2}-r_{M}^{2}
\end{array}\right]
$$

Using (47), (46) is represented as

$$
\begin{aligned}
J=\left[\mathbf{G} \breve{\mathbf{x}}_{0}-0.5\left(\breve{\mathbf{x}}_{0}^{T} \breve{\mathbf{x}}_{0}\right) \mathbf{1}_{M}-\mathbf{h}\right]^{T} \mathbf{W} \\
\cdot\left[\mathbf{G} \breve{\mathbf{x}}_{0}-0.5\left(\breve{\mathbf{x}}_{0}^{T} \breve{\mathbf{x}}_{0}\right) \mathbf{1}_{M}-\mathbf{h}\right]
\end{aligned}
$$

where

$$
\mathbf{W}=4\left(\frac{1}{2} \mathbf{I}_{M}-\frac{M-2}{2 M^{2}} \mathbf{1}_{M} \mathbf{1}_{M}^{T}\right)
$$

which is a weighted LS cost function with weighting matrix $\mathbf{W}$.

By using (31), we have proved in Appendix A that unbiased MS position estimation is attained via minimizing (48) for sufficiently small range errors. The required derivatives of $J$ for variance analysis are computed as (see Appendix B)

$$
\begin{aligned}
& \left.E\left[\left(\frac{\partial J}{\partial \breve{\mathbf{x}}_{0}}\right)\left(\frac{\partial J}{\partial \breve{\mathbf{x}}_{0}}\right)^{T}\right]\right|_{\breve{\mathbf{x}}_{0}=\mathbf{x}_{0}} \\
& \approx 4\left(\mathbf{G}^{T}-\mathbf{x}_{0} \mathbf{1}_{M}^{T}\right) \mathbf{W}\left(\mathbf{d d}^{T} \odot \mathbf{C}_{\mathbf{n}}\right) \mathbf{W}\left(\mathbf{G}-\mathbf{1}_{M} \mathbf{x}_{0}^{T}\right)
\end{aligned}
$$

where $\mathbf{C}_{\mathbf{n}}$ represents the covariance matrix of the vector of range errors at the $M \mathrm{BSs}$, which are denoted by $\mathbf{n}$, and $\odot$ is the Schur or element-by-element product, with $\mathbf{d}=\left[\begin{array}{llll}d_{1} & d_{2} & \cdots & d_{M}\end{array}\right]^{T}$ and $\mathbf{n}=\left[\begin{array}{llll}n_{1} & n_{2} & \cdots & n_{M}\end{array}\right]^{T}$, whose elements are given by (4), and

$$
\left.E\left[\frac{\partial^{2} J}{\partial \breve{\mathbf{x}}_{0} \partial \breve{\mathbf{x}}_{0}^{T}}\right]\right|_{\breve{\mathbf{x}}_{0}=\mathbf{x}_{0}} \approx 2\left(\mathbf{G}^{T}-\mathbf{x}_{0} \mathbf{1}_{M}^{T}\right) \mathbf{W}\left(\mathbf{G}-\mathbf{1}_{M} \mathbf{x}_{0}^{T}\right)
$$

Finally, substituting (49) and (50) into (34) gives the covariance matrix $\mathbf{C}_{\mathbf{x}_{\mathbf{0}}}$ for the proposed method. The variances of $\hat{x}_{0}$ and $\hat{y}_{0}$ are then obtained from the diagonal elements of $\mathbf{C}_{\mathbf{x}_{0}}$.

Finally, the relationships between the MDS, LSC, two-step WLS, LLS, and QLS estimators, which give globally convergence solutions, will be illustrated as follows. If we substitute W with $\mathbf{I}_{M}$ and consider $\breve{\mathbf{x}}_{0}^{T} \breve{\mathbf{x}}_{0}$ as an extra variable in (48), the resultant cost function corresponds to the LSC estimator, whose solution can be obtained easily via standard LS technique, although the known relation between the extra variable and $\breve{\mathbf{x}}_{0}$ is not utilized. When $\mathbf{W}$ is substituted with the optimum weighting matrix [22], (48) represents the cost function of the two-step WLS method, assuming that the relation between the extra variable and $\breve{\mathbf{x}}_{0}$ can be explicitly exploited in the second step of the algorithm. Replacing $\mathbf{W}$ with $\mathbf{I}_{M}$ and removing all terms with $\breve{\mathbf{x}}_{0}^{T} \breve{\mathbf{x}}_{0}$ in (48), the resultant cost function corresponds to the LLS method, and interestingly, it can be shown that the LSC and LLS estimators are equivalent. Moreover, the cost function for the QLS method is given by (48) with the substitution of $\mathbf{W}=\mathbf{I}_{M}$ only. It is noteworthy that since the LSC, two-step WLS, LLS, and QLS estimators can be interpreted using (48), we are able to use the derived bias and variance formulae to produce their performance as well.

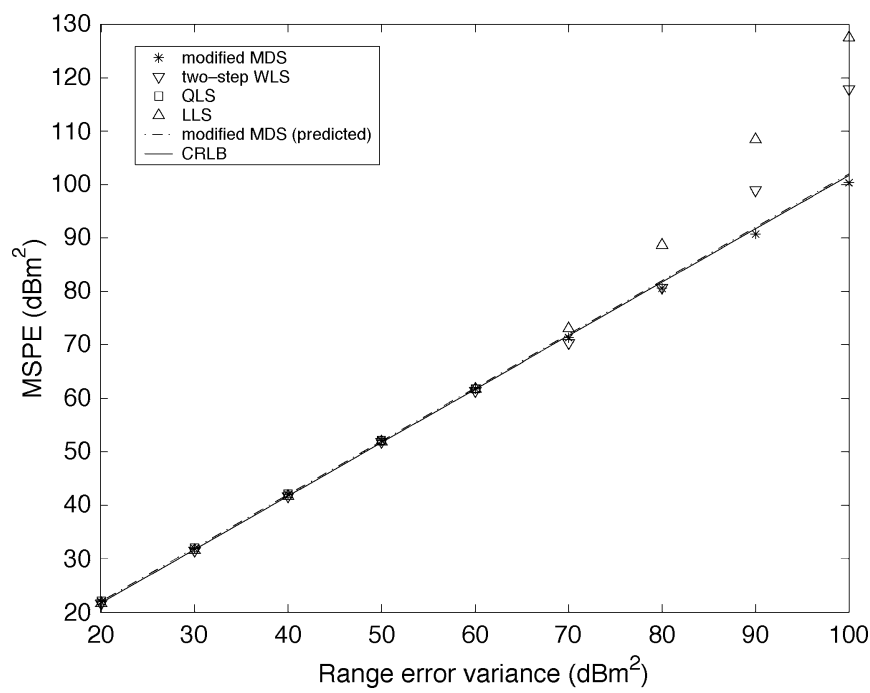

Fig. 1. Mean square position errors for three-BS geometry without NLOS errors at $\left[x_{0}, y_{0}\right]=[1000,2000] \mathrm{m}$.

\section{NUMERICAL EXAMPLES}

This section contains MATLAB simulation results to corroborate the theoretical development and to evaluate the performance of the proposed TOA-based location algorithm by comparing with the LLS, QLS [21], and two-step WLS algorithms, where the latter is easily adapted from [22], where TDOA-based location is addressed. The LSC method [20] was not included since it is equivalent to the LLS estimator. Notice that the two-step WLS algorithm expects to outperform the LSC/LLS method because [22] has been shown to be superior to the SI approach [18], [19]. The range errors $\left\{n_{i}\right\}$ were zero-mean white Gaussian processes with identical variances. All results were averages of 1000 independent runs.

Fig. 1 plots the mean square position errors (MSPEs) of the modified MDS, LLS, QLS, and two-step WLS methods versus range error variance. The theoretical performance of the proposed method and the corresponding Cramér-Rao lower bound (CRLB) [31] (See Appendix C) were also included. The MSPE was defined as $E\left[\left(x_{0}-\hat{x}_{0}\right)^{2}+\left(y_{0}-\hat{y}_{0}\right)^{2}\right]$. We considered a three-BS geometry with coordinates $[0,0] \mathrm{m}$, $[3000 \sqrt{3}, 3000] \mathrm{m}$ and $[0,6000] \mathrm{m}$, while the MS position was fixed at $\left[x_{0}, y_{0}\right]=[1000,2000] \mathrm{m}$, and notice that the centroid requirement was fulfilled by simple translation in the coordinate system. It can be seen that the proposed algorithm performed similarly with the two-step WLS, QLS, and LLS methods, which gave large estimation errors or threshold effects [31] at 90,70 , and $80 \mathrm{dBm}^{2}$, respectively, which indicated that the MDS method was more robust than the other algorithms as it had a larger operation range. One possible reason for the robustness of the proposed estimator is that nonzero-mean disturbances in $\left\{d_{i j}^{2}\right\}$ of (15) are allowed in the classical MDS formulation, whereas the remaining methods assume zero-mean $\left\{\epsilon_{i}^{2}\right\}$ in (36), which is invalid for sufficiently large range errors, in applying linearization to (4) in their algorithm development [20]-[22]. It is noteworthy that the mobile location estimation problem is nonlinear, and hence, all positioning algorithms, including the MDS method, will suffer from the threshold phenomenon or known as occurrence of outliers, for sufficiently 


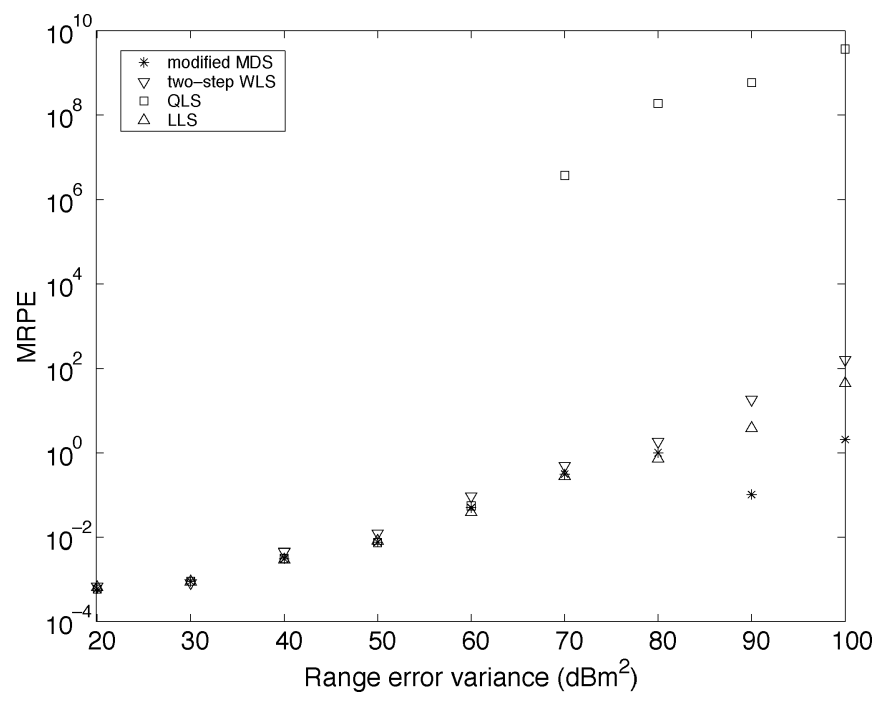

Fig. 2. Mean relative position errors for three-BS geometry without NLOS errors at $\left[x_{0}, y_{0}\right]=[1000,2000] \mathrm{m}$.

large noise conditions. Moreover, there was good agreement between the measured MSPE and theoretical variance of the proposed algorithm, where both were close to the CRLB. Notice that the MSPEs of the QLS estimator with range errors greater than $60 \mathrm{dBm}^{2}$ were too large to be included in Fig. 1. The corresponding mean relative position errors (MRPEs) of the investigated methods, which was a measure of bias and were defined as $\sqrt{\left[\left(E\left[\hat{x}_{0}\right]-x_{0}\right) / x_{0}\right]^{2}+\left[\left(E\left[\hat{y}_{0}\right]-y_{0}\right) / y_{0}\right]^{2}}$, are shown in Fig. 2. It is observed that the algorithms had comparable MRPEs for sufficiently small range errors, and this implied that they had similar empirical biases that were close to zero for such conditions. For larger noise environment, the proposed algorithm obviously had similar measured MRPEs with those of the two-step WLS and LLS techniques. The QLS algorithm had MRPE values over $10^{6}$, which indicated that its position estimates were very far from the true position, and this observation also conformed to Fig. 1. Furthermore, the number of floating-point operations (FLOPS) in computing a position estimate using the MDS, LLS, QLS, and two-step WLS algorithms were $1530,109,2869$, and 1200, respectively, which implied that the computational complexity of the proposed algorithm was moderate.

Fig. 3 plots the MSPEs of all the algorithms when the MS position was randomly chosen in an area bounded by $[0,0]$, $[3000 \sqrt{3}, 3000],[-3000 \sqrt{3}, 3000]$, and $[0,6000] \mathrm{m}$ for each trial. We used the three-BS geometry as in the previous test, which implied that approximately half of the MS positions would be inside the region bounded by the BSs, and the remaining would be outside this region. As a result, the estimation performance of the methods was basically different at each trial because it is well known [15] that the positioning accuracy varies with the relative MS and BS geometry. Nevertheless, we observe similar findings, as in Fig. 1; in particular, the modified MDS method was robust for larger range errors.

Fig. 4 shows the MSPEs of the algorithms versus number of BSs when the range error variance was kept at $40 \mathrm{dBm}^{2}$ with $\left[x_{0}, y_{0}\right]=[1000,2000] \mathrm{m}$. The minimum BS number was 3 , the corresponding geometry was identical to the previous test, and the BSs with coordinates $[-3000 \sqrt{3}, 3000]$,

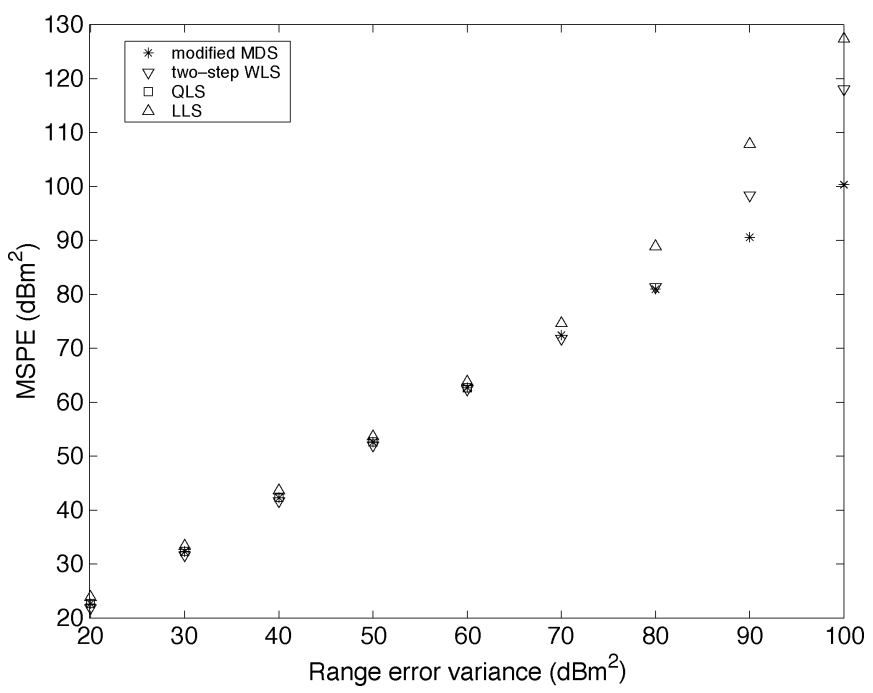

Fig. 3. Mean square position errors for 3-BS geometry with random MS positions.

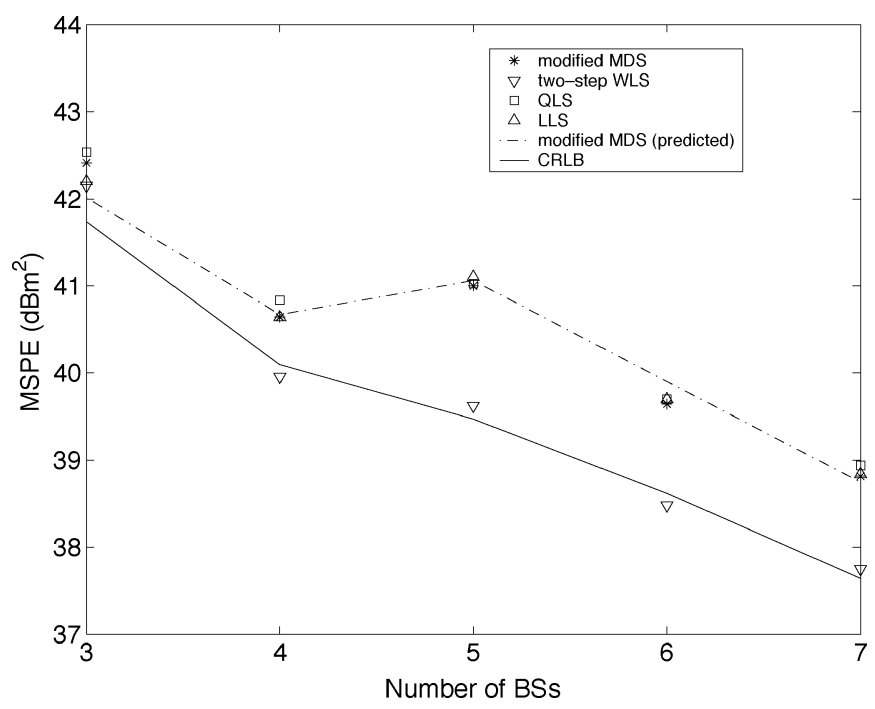

Fig. 4. Mean square position errors versus number of BSs under $40 \mathrm{dBm}^{2}$ measurement errors at $\left[x_{0}, y_{0}\right]=[1000,2000] \mathrm{m}$.

$[-3000 \sqrt{3},-3000], \quad[0,-6000]$, and $[3000 \sqrt{3},-3000] \mathrm{m}$ were then added successively. Notice that variations in the BS number also produces changes in the BS geometry, and subsequently, the positioning accuracy varies as well [15]. In the figure, it is seen that the estimation accuracy of all methods generally increased with the number of BSs. The proposed algorithm performed similarly with the LLS and QLS methods but was inferior to the two-step WLS estimator, which met the CRLB, by around $1 \mathrm{dBm}^{2}$. The optimality of the two-step WLS method is due to the use of the optimum weighting matrix in (48), whereas the remaining algorithms do not. Furthermore, the agreement between the measured MSPEs and derived performance of the MDS estimator was again illustrated.

The first test was repeated for positive-mean TOA errors to simulate the effect of NLOS propagation, and the range measurements were now modeled as [32]

$$
r_{i}=d_{i}+n_{i}+N m_{i}
$$

where $N$ was the maximum allowable error introduced by NLOS, and $m_{i}$ and $i=1,2, \cdots, M$ were independent uni- 
formly distributed random numbers ranged from 0 to 1 . In this experiment, $N$ was set to be $500 \mathrm{~m}$, and the results are shown in Figs. 5 and 6. Again, it can be seen that the proposed algorithm performed similarly with other algorithms but was more robust at larger range error conditions. Note that the zero-mean disturbances $\left\{n_{i}\right\}$ dominated the NLOS error, whose maximum value was $500 \mathrm{~m}$, when the range error variance was greater than $50 \mathrm{dBm}^{2}$, and thus, the NLOS effect was negligible for larger $\left\{n_{i}\right\}$.

\section{CONCLUSIONS AND FUTURE WORKS}

An accurate and computationally simple mobile positioning algorithm using time-of-arrival measurements has been proposed. The algorithm development is based on modifying the classical multidimensional scaling (MDS) technique. A variance analysis formula for general optimization problems is developed to produce the theoretical performance of the modified MDS method, which is verified via computer simulations for sufficiently small error conditions.

The proposed method can be employed for positioning systems using received signal strength measurements, which is a straightforward extension. Future works also include investigation of estimation performance improvement for the classical MDS algorithm, if possible, via utilizing a proper weighting function in (35). In addition, we will explore the possibility of utilizing MDS in time-difference-of-arrival based location as well as other signal processing applications.

\section{APPENDIX A}

Taking the expected value on both sides of (31) and rearranging the resultant equation yields an expression for the bias of the estimated MS position:

$$
E\left[\hat{\mathbf{x}}_{0}\right]-\mathbf{x}_{0} \approx-\left.E\left[\frac{\partial^{2} J}{\partial \breve{\mathbf{x}}_{0} \partial \breve{\mathbf{x}}_{0}^{T}}\right]^{-1} E\left[\frac{\partial J}{\partial \breve{\mathbf{x}}_{0}}\right]\right|_{\breve{\mathbf{x}}_{0}=\mathbf{x}_{0}} .
$$

Differentiating (48) with respect to $\breve{\mathbf{x}}_{0}$, we get

$$
\frac{\partial J}{\partial \breve{\mathbf{x}}_{0}}=2\left(\mathbf{G}^{T}-\breve{\mathbf{x}}_{0} \mathbf{1}_{M}^{T}\right)\left[\mathbf{G}_{\mathbf{x}_{0}}-0.5\left(\breve{\mathbf{x}}_{0}^{T} \breve{\mathbf{x}}_{0}\right) \mathbf{1}_{M}-\mathbf{h}\right] .
$$

Assuming that the disturbance due to the TOA measurements is sufficiently small such that $\left\{n_{i}^{2}\right\}$ can be ignored, the derivative of $J$ evaluated at the true source position $\mathbf{x}_{0}$ becomes

$$
\left.\frac{\partial J}{\partial \breve{\mathbf{x}}_{0}}\right|_{\breve{\mathbf{x}}_{0}=\mathbf{x}_{0}} \approx 2\left(\mathbf{G}^{T}-\mathbf{x}_{0} \mathbf{1}_{M}^{T}\right) \mathbf{W}(\mathbf{d} \odot \mathbf{n}) .
$$

We take the expected value on both sides of (A.3) and then apply the fact that $E[\mathbf{n}]=\mathbf{0}$ to obtain

$$
\left.E\left[\frac{\partial J}{\partial \breve{\mathbf{x}}_{0}}\right]\right|_{\breve{\mathbf{x}}_{0}=\mathbf{x}_{0}} \approx 2\left(\mathbf{G}^{T}-\mathbf{x}_{0} \mathbf{1}_{M}^{T}\right) \mathbf{W} E[\mathbf{d} \odot \mathbf{n}]=\mathbf{0} .
$$

Substituting (A.4) into (A.1) implies

$$
E\left[\hat{\mathbf{x}}_{0}\right] \approx \mathbf{x}_{0}
$$

which illustrates the unbiasedness of the proposed algorithm when the first matrix in (A.1) is nonsingular.

\section{APPENDIX B}

Multiplying (A.3) by its transpose yields

$$
\begin{aligned}
& \left.\left(\frac{\partial J}{\partial \breve{\mathbf{x}}_{0}}\right)\left(\frac{\partial J}{\partial \breve{\mathbf{x}}_{0}}\right)^{T}\right|_{\breve{\mathbf{x}}_{0}=\mathbf{x}_{0}} \\
& \approx 4\left(\mathbf{G}^{T}-\mathbf{x}_{0} \mathbf{1}_{M}^{T}\right) \mathbf{W}(\mathbf{d} \odot \mathbf{n})(\mathbf{d} \odot \mathbf{n})^{T} \mathbf{W}\left(\mathbf{G}-\mathbf{1}_{M} \mathbf{x}_{0}^{T}\right) .
\end{aligned}
$$

By taking the expected value of (A.6), (49) is obtained.

Differentiating (A.2) with respect to $\breve{x}_{0}$, the first variable in $\breve{\mathbf{x}}_{0}$, and with the use of product rule, we get

$$
\begin{aligned}
\frac{\partial}{\partial \breve{x}_{0}}\left(\frac{\partial J}{\partial \breve{\mathbf{x}}_{0}}\right) & \\
= & 2\left(\mathbf{G}^{T}-\breve{\mathbf{x}}_{0} \mathbf{1}_{M}^{T}\right) \mathbf{W} \frac{\partial}{\partial \breve{x}_{0}}\left[\mathbf{G} \breve{\mathbf{x}}_{0}-0.5\left(\breve{\mathbf{x}}_{0}^{T} \breve{\mathbf{x}}_{0}\right) \mathbf{1}_{M}-\mathbf{h}\right] \\
& +2\left[\frac{\partial}{\partial \breve{x}_{0}}\left(\mathbf{G}^{T}-\breve{\mathbf{x}}_{0} \mathbf{1}_{M}^{T}\right)\right] \mathbf{W}\left[\mathbf{G} \breve{\mathbf{x}}_{0}-0.5\left(\breve{\mathbf{x}}_{0}^{T} \breve{\mathbf{x}}_{0}\right) \mathbf{1}_{M}-\mathbf{h}\right] \\
= & 2\left(\mathbf{G}^{T}-\breve{\mathbf{x}}_{0} \mathbf{1}_{M}^{T}\right) \mathbf{W}\left[\mathbf{G}\left[\begin{array}{l}
1 \\
0
\end{array}\right]-\mathbf{1}_{M} \breve{x}_{0}\right] \\
& +2\left[\frac{\partial}{\partial \breve{x}_{0}}\left(\mathbf{G}^{T}-\breve{\mathbf{x}}_{0} \mathbf{1}_{M}^{T}\right)\right] \mathbf{W}\left[\mathbf{G} \breve{\mathbf{x}}_{0}-0.5\left(\breve{\mathbf{x}}_{0}^{T} \breve{\mathbf{x}}_{0}\right) \mathbf{1}_{M}-\mathbf{h}\right]
\end{aligned}
$$

Ignoring the terms of $\left\{n_{i}^{2}\right\}$ again, the value of (A.7) computed at $\mathbf{x}_{0}$ is

$$
\begin{array}{r}
\left.\frac{\partial}{\partial \breve{x}_{0}}\left(\frac{\partial J}{\partial \breve{\mathbf{x}}_{0}}\right)\right|_{\breve{\mathbf{x}}_{0}=\mathbf{x}_{0}}=2\left(\mathbf{G}^{T}-\mathbf{x}_{0} \mathbf{1}_{M}^{T}\right) \mathbf{W}\left[\mathbf{G}\left[\begin{array}{l}
1 \\
0
\end{array}\right]-\mathbf{1}_{M} x_{0}\right] \\
+\left.2\left[\frac{\partial}{\partial \breve{x}_{0}}\left(\mathbf{G}^{T}-\breve{\mathbf{x}}_{0} \mathbf{1}_{M}^{T}\right)\right]\right|_{\breve{\mathbf{x}}_{0}=\mathbf{x}_{0}} \mathbf{W}(\mathbf{d} \odot \mathbf{n})
\end{array}
$$

Taking the expected value on both sides of (A.8) and applying $E[\mathbf{n}]=\mathbf{0}$ gives

$\left.E\left[\frac{\partial}{\partial \breve{x}_{0}}\left(\frac{\partial J}{\partial \breve{\mathbf{x}}_{0}}\right)\right]\right|_{\breve{\mathbf{x}}_{0}=\mathbf{x}_{0}} \approx 2\left(\mathbf{G}^{T}-\mathbf{x}_{0} \mathbf{1}_{M}^{T}\right) \mathbf{W}\left[\mathbf{G}\left[\begin{array}{l}1 \\ 0\end{array}\right]-\mathbf{1}_{M} x_{0}\right]$.

Similarly, repeating the derivations in (A.7)-(A.9) for the second variable $\breve{y}_{0}$, we obtain

$\left.E\left[\frac{\partial}{\partial \breve{y}_{0}}\left(\frac{\partial J}{\partial \breve{\mathbf{x}}_{0}}\right)\right]\right|_{\breve{\mathbf{x}}_{0}=\mathbf{x}_{0}} \approx 2\left(\mathbf{G}^{T}-\mathbf{x}_{0} \mathbf{1}_{M}^{T}\right) \mathbf{W}\left[\mathbf{G}\left[\begin{array}{l}0 \\ 1\end{array}\right]-\mathbf{1}_{M} y_{0}\right]$.

Since

$\left.E\left[\frac{\partial^{2} J}{\partial \breve{\mathbf{x}}_{0} \partial \breve{\mathbf{x}}_{0}^{T}}\right]\right|_{\breve{\mathbf{x}}_{0}=\mathbf{x}_{0}}$
$=\left[\left.\left.E\left[\frac{\partial}{\partial \breve{x}_{0}}\left(\frac{\partial J}{\partial \breve{\mathbf{x}}_{0}}\right)\right]\right|_{\breve{\mathbf{x}}_{0}=\mathbf{x}_{0}} E\left[\frac{\partial}{\partial \breve{y}_{0}}\left(\frac{\partial J}{\partial \breve{\mathbf{x}}_{0}}\right)\right]\right|_{\breve{\mathbf{x}}_{0}=\mathbf{x}_{0}}\right]$

substituting (A.9) and (A.10) into (A.11) yields (50).

\section{APPENDIX C}

The CRLB gives a lower bound on variance attainable by any unbiased estimators. To determine the CRLB for mobile 
location, we first define $\mathbf{r}=\left[\begin{array}{llll}r_{1} & r_{2} & \cdots & r_{M}\end{array}\right]^{T}$, and its expected value is easily shown to be

$$
E[\mathbf{r}]=\overline{\mathbf{r}}=\left[\begin{array}{c}
\sqrt{\left(x_{0}-x_{1}\right)^{2}+\left(y_{0}-y_{1}\right)^{2}} \\
\sqrt{\left(x_{0}-x_{2}\right)^{2}+\left(y_{0}-y_{2}\right)^{2}} \\
\vdots \\
\sqrt{\left(x_{0}-x_{M}\right)^{2}+\left(y_{0}-y_{M}\right)^{2}}
\end{array}\right] .
$$

According to [31], when the TOA measurement errors are Gaussian distributed, the Fisher information matrix for the MS location $\mathbf{x}_{0}$ is given by

$$
\mathbf{I}\left(\mathbf{x}_{0}\right)=\left[\begin{array}{ll}
\left(\frac{\partial \overline{\mathbf{r}}}{\partial x_{0}}\right)^{T} \mathbf{C}_{\mathbf{n}}^{-1}\left(\frac{\partial \overline{\mathbf{r}}}{\partial x_{0}}\right) & \left(\frac{\partial \overline{\mathbf{r}}}{\partial x_{0}}\right)^{T} \mathbf{C}_{\mathbf{n}}^{-1}\left(\frac{\partial \overline{\mathbf{r}}}{\partial y_{0}}\right) \\
\left(\frac{\partial \overline{\mathbf{r}}}{\partial y_{0}}\right)^{T} \mathbf{C}_{\mathbf{n}}^{-1}\left(\frac{\partial \overline{\mathbf{r}}}{\partial x_{0}}\right) & \left(\frac{\partial \overline{\mathbf{r}}}{\partial y_{0}}\right)^{T} \mathbf{C}_{\mathbf{n}}^{-1}\left(\frac{\partial \overline{\mathbf{r}}}{\partial y_{0}}\right)
\end{array}\right]
$$

where the $i$ th elements of $\left(\partial \overline{\mathbf{r}} / \partial x_{0}\right)$ and $\left(\partial \overline{\mathbf{r}} / \partial y_{0}\right)$ are computed as

$$
\left[\frac{\partial \overline{\mathbf{r}}}{\partial x_{0}}\right]_{i}=\frac{\left(x_{0}-x_{i}\right)}{\sqrt{\left(x_{0}-x_{i}\right)^{2}+\left(y_{0}-y_{i}\right)^{2}}}
$$

and

$$
\left[\frac{\partial \overline{\mathbf{r}}}{\partial y_{0}}\right]_{i}=\frac{\left(y_{0}-y_{i}\right)}{\sqrt{\left(x_{0}-x_{i}\right)^{2}+\left(y_{0}-y_{i}\right)^{2}}}, \quad i=1,2, \cdots, M
$$

The CRLBs for $x_{0}$ and $y_{0}$ are given by the first and second diagonal elements of $\mathbf{I}^{-1}\left(\mathbf{x}_{0}\right)$, respectively.

\section{ACKNOWLEDGMENT}

The authors would like to thank the anonymous reviewers for their careful reading and constructive suggestions that improved the quality of this paper.

\section{REFERENCES}

[1] Revision of the Commissions Rules to Ensure Compatibility With Enhanced 911 Emergency Calling Systems, RM-8143, CC Docket no. 94-102, Jul. 26, 1996.

[2] C. Drane, M. Macnaughtan, and C. Scott, "Positioning GSM telephones," IEEE Commun. Mag., pp. 46-54, Apr. 1998.

[3] H. Koshima and J. Hosen, "Personal locator services emerge," IEEE Spectrum, pp. 41-48, Feb. 2000.

[4] Y. Zhao, "Mobile phone location determination and its impact on intelligent transport systems," IEEE Trans. Intell. Transp. Syst., vol. 1, pp. 55-64, Mar. 2000.

[5] D. Porcino, "Performance of a OTDOA-IPDL positioning receiver for 3G-FDD mode," in Proc. Int. Conf. 3 G Mobile Commun. Technol., 2001, pp. 221-225.

[6] J. J. Caffery Jr., Wireless Location in CDMA Cellular Radio Systems. Boston, MA: Kluwer, 2000.

[7] S. S. Soliman and C. E. Wheatley, "Geolocation technologies and applications for third generation wireless," Wireless Commun. Mobile Comput., vol. 2, pp. 229-251, May 2002.

[8] J. Vidal, M. Najar, and R. Jativa, "High resolution time-of-arrival detection for wireless positioning systems," in Proc. IEEE Veh. Technol. Conf., vol. 4, Sep. 2002, pp. 2283-2287.

[9] J. Riba and A. Urruela, "A robust multipath mitigation technique for time-of-arrival estimation," in Proc. IEEE Veh. Technol. Conf., vol. 4 , Sep. 2002, pp. 2263-2267.

[10] S. Al-Jazzar, J. J. Caffery Jr., and H.-R. You, "A scattering model based approach to NLOS mitigation in TOA location systems," in Proc. IEEE Veh. Technol. Conf., vol. 2, May 2002, pp. 861-865.
[11] M. P. Wylie-Green and S. S. Wang, "Robust range estimation in the presence of the nonline-of-sight error," in Proc. IEEE Veh. Technol. Conf., vol. 1, Sep. 2001, pp. 101-105.

[12] N. Patwari, A. O. Hero III, M. Perkins, N. S. Correal, and R. J. O'Dea "Relative location estimation in wireless sensor networks," IEEE Trans. Signal Process., vol. 51, no. 8, pp. 2137-2148, Aug. 2003.

[13] R. J. Fontana and S. J. Gunderson, "Ultra-wideband precision asset location system," in Proc. IEEE Conf. Ultra Wideband Syst. Technol., May 2002, pp. 147-150.

[14] D. J. Torrieri, "Statistical theory of passive location systems," IEEE Trans. Aerosp. Electron. Syst., vol. AES-20, pp. 183-197, Mar. 1984.

[15] M. A. Spirito, "On the accuracy of cellular mobile station location estimation," IEEE Trans. Veh. Technol., vol. 50, pp. 674-685, May 2001.

[16] J. J. Caffery Jr. and G. L. Stuber, "Subscriber location in CDMA cellular networks," IEEE Trans. Veh. Technol., vol. 47, pp. 406-416, May 1998.

[17] M. Hellebrandt, R. Mathar, and M. Scheibenbogen, "Estimating position and velocity of mobiles in a cellular radio network," IEEE Trans. Veh. Technol., vol. 46, no. 1, pp. 65-71, Feb. 1997.

[18] J. O. Smith and J. S. Abel, "Closed-form least-squares source location estimation from range-difference measurements," IEEE Trans. Acoust., Speech, Signal Process., vol. ASSP-35, pp. 1661-1669, Dec. 1987.

[19] B. Friedlander, "A passive localization algorithm and its accuracy analysis," IEEE J. Ocean. Eng., vol. OE-12, pp. 234-245, Jan. 1987.

[20] J. C. Chen, R. E. Hudson, and K. Yao, "Maximum-likelihood source localization and unknown sensor location estimation for wideband signals in the near field," IEEE Trans. Signal Process., vol. 50, no. 8, pp. 1843-1854, Aug. 2002.

[21] A. J. Fenwick, "Algorithms for position fixing using pulse arrival times," Proc. Inst. Elect. Eng., Radar, Sonar Navig., vol. 146, no. 4, pp. 208-212, Aug. 1999.

[22] Y. T. Chan and K. C. Ho, "A simple and efficient estimator for hyperbolic location,” IEEE Trans. Signal Process., vol. 42, pp. 1905-1915, Aug. 1994.

[23] S. T. Birchfield, "Geometric microphone array calibration by multidimensional scaling," in Proc. IEEE Int. Conf. Acoust., Speech, Signal Process., vol. 5, Hong Kong, Apr. 2003, pp. 157-160.

[24] Y. Shang, W. Ruml, Y. Zhang, and M. P. J. Fromherz, "Localization from mere connectivity," in Proc. ACM Int. Symp. Mobile Ad Hoc Networking Comput., 2003, pp. 201-212.

[25] T. F. Cox and M. A. A. Cox, Multidimensional Scaling. Boca Raton, FL: Chapman Hall/CRC, 2001.

[26] I. Borg and P. Groenen, Modern Multidimensional Scaling: Theory and Applications. New York: Springer, 1997.

[27] H. E. A. Tinsley and S. D. Brown, Handbook of Applied Multivariate Statistics and Mathematical Modeling. San Diego, CA: Academic, 2000.

[28] G. Young and A. S. Householder, "Discussion of a set of points in terms of their mutual distances," Psychometrika, vol. 3, no. 1, pp. 19-22, Mar. 1938.

[29] W. S. Torgerson, "Multidimensional scaling: I. Theory and method," Psychometrika, vol. 17, no. 4, pp. 401-419, Dec. 1952.

[30] J. C. Gower, "Some distance properties of latent root and vector methods used in multivariate analysis," Biometrika, vol. 53, pp. 325-338, 1966.

[31] S. M. Kay, Fundamentals of Statistical Signal Processing: Estimation Theory. Englewood Cliffs, NJ: Prentice-Hall, 1993.

[32] X. Wang, Z. Wang, and B. O'Dea, "A TOA-based location algorithm reducing the errors due to nonline-of-sight (NLOS) propagation," IEEE Trans. Veh. Technol., vol. 52, no. 1, pp. 112-116, Jan. 2003.

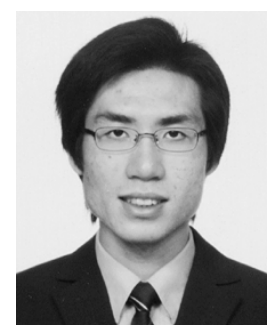

K. W. Cheung was born in Hong Kong. He received the B.Eng. degree with First Class Honors in electrical and electronic engineering from Imperial College of Science, Technology, and Medicine, University of London, London, U.K., in 2001. From October to November 2001, he was a Research Assistan with the Department of Computer Engineering and Information Technology, the City University of Hong Kong, where he is currently pursuing an M.Phil. degree.

His research interests are in array signal processing and developing efficient methods in radiolocation for mobile terminals.

Mr. Cheung is an Associate Member of both Institution of Electrical Engineers in the U.K. and the Hong Kong Institution of Engineers. 


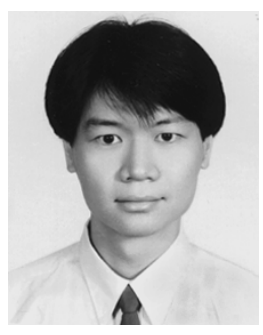

H. C. So (M'95) was born in Hong Kong. He received the B.Eng. degree in electronic engineering from City Polytechnic of Hong Kong in 1990 and the Ph.D. degree in electronic engineering from The Chinese University of Hong Kong in 1995.

From 1990 to 1991, he was an Electronic Engineer with the Research and Development Division of Everex Systems Engineering Ltd., Hong Kong. He then worked as a Post-Doctoral Fellow at The Chinese University of Hong Kong and was responsible for devising and analyzing efficient algorithms for geolocation. From 1996 to 1999, he was a Research Assistant Professor with the Department of Electronic Engineering, City University of Hong Kong (CUHK). Currently, he is an Assistant Professor with the Department of Computer Engineering and Information Technology, CUHK. His research interests include adaptive filter theory, detection and estimation, wavelet transforms, and signal processing for communications and multimedia. 